\title{
Shen-Fu Injection Preconditioning Inhibits Myocardial Ischemia-Reperfusion Injury in Diabetic Rats: Activation of eNOS via the PI3K/Akt Pathway
}

\author{
Yang Wu, ${ }^{1}$ Zhong-yuan Xia, ${ }^{1}$ Qing-tao Meng, ${ }^{1}$ Jie Zhu, ${ }^{2}$ Shaoqing Lei, ${ }^{3}$ Jinjin Xu, ${ }^{1}$ \\ and Juan Dou ${ }^{4}$ \\ ${ }^{1}$ Department of Anesthesiology, Renmin Hospital of Wuhan University, Wuhan, Hubei 430060, China \\ ${ }^{2}$ Department of Gastroenterology, Renmin Hospital of Wuhan University, Wuhan, Hubei 430060, China \\ ${ }^{3}$ Department of Pharmacology, School of Medicine, Wuhan University, Donghu Road, Wuhan430071, China \\ ${ }^{4}$ Department of Cardiovascular Surgery, Renmin Hospital of Wuhan University, Wuhan, Hubei430060, China
}

Correspondence should be addressed to Zhong-yuan Xia, xiazhongyuanmz@yahoo.cn

Received 19 August 2010; Accepted 22 October 2010

Academic Editor: Monica Fedele

Copyright (C) 2011 Yang Wu et al. This is an open access article distributed under the Creative Commons Attribution License, which permits unrestricted use, distribution, and reproduction in any medium, provided the original work is properly cited.

\begin{abstract}
The aim of this paper is to investigate whether Shen-fu injection (SFI), a traditional Chinese medicine, could attenuate myocardial ischemia-reperfusion (MI/R) injury in diabetes. Streptozotocin-induced diabetic rats were randomly assigned to the Sham, I/R, SFI preconditioning, and SFI plus wortmannin (a phosphatidylinositol 3-kinase inhibitor) groups. After the treatment, hearts were subjected to $30 \mathrm{~min}$ of coronary artery occlusion and $2 \mathrm{~h}$ reperfusion except the Sham group. Myocardial infarct size and cardiomyocytes apoptosis were increased significantly in MI/R group as compared with the Sham group. SFI preconditioning significantly decreased infarct size, apoptosis, caspase-3 protein expression, MDA level in myocardial tissues, and plasma level of CK and LDH but increased p-Akt, p-eNOS, bcl-2 protein expression, and SOD activity compared to I/R group. Moreover, SFIinduced cardioprotection was abolished by wortmannin. We conclude that SFI preconditioning protects diabetic hearts from I/R injury via PI3K/Akt-dependent pathway.
\end{abstract}

\section{Introduction}

Epidemiological and pathological data show that diabetes is a major risk for cardiovascular morbidity and mortality $[1,2]$. Ischemic coronary artery disease is responsible for three-quarters of diabetes-related death [3]. Approximately $50 \%$ of diabetic patients die 5 years after a myocardial infarction, double the rate found in nondiabetic patients $[4,5]$. The poor prognosis may be at least in part because of an increase in the myocardial injury in response to ischemia and reperfusion [5].

Shen-Fu injection (SFI), an extract of traditional Chinese herbs, has been routinely used in treating cardiac diseases for a long time in China. We have previously demonstrated that SFI could attenuate myocardial ischemia-reperfusion (MI/R) injury and enhance postoperative myocardial functional recovery in patients undergoing cardiopulmonary bypass heart operations [6]. The beneficial effects of SFI may attribute to alleviating the cell injuries during ischemia reperfusion. Moreover, the mechanism of SFI's cardioprotection remains to be elucidated. The effects of SFI preconditioning on diabetic rats following ischemia-reperfusion injury is not well understood.

In the present study, we aimed to investigate whether SFI protects diabetic rats from I/R injury and, more importantly, to explore the underlying mechanisms.

\section{Materials and Methods}

2.1. Experimental Animals. The experimental protocol used in this study was reviewed and approved by the Animal Care and Use Committee of Wuhan University and in accordance with the National Institutes of Health guidelines for the use of experimental animals. Male Sprague-Dawley (SD) rats weighing $240 \mathrm{~g}$ to $280 \mathrm{~g}$ were provided by the Experimental 
Animal Center of Wuhan University. All animals were allowed free access to food and water and maintained at 2224 degree Celsius under a cycle of $12 \mathrm{~h}$ : 12h light-dark.

2.2. Drugs and Reagents. Streptozotocin (STZ), triphenyltetrazolium chloride (TTC), Evans blue (EB), and wortmannin were purchased from Sigma (St. Louis, MO, USA). Shen-fu injection (contains $0.9 \mathrm{mg}$ ginsenosides and $0.1 \mathrm{mg}$ aconite alkaloid per milliliter) was produced by Ya'an Sanjiu Pharmaceutical Co., Ltd., China.

2.3. Induction of Diabetes. Experimental diabetes was induced in male SD rats by intravenous injection of STZ dissolved in $0.1 \mathrm{~mol} / \mathrm{L}$ citrate buffer $(\mathrm{pH} 4.5)$ at a dose of $65 \mathrm{mg} / \mathrm{kg}$. Three days after STZ injection, hyperglycemia was documented by measuring the glucose content of tail vein blood with OneTouch glucometer (Johnson and Johnson, USA). Rats with blood glucose concentrations $\geq$ $16.7 \mathrm{mmol} / \mathrm{L}$ were considered to be diabetic.

2.4. Surgical Preparations. All animals were anesthetized with IP injection of pentobarbital sodium $(50 \mathrm{mg} / \mathrm{kg})$ and ventilated with room air. A cannula was inserted into the left femoral vein for administration of drugs and into the left carotid artery for measurement of blood pressure, respectively. Limb lead II of the ECG was used to measure the heart rate. A fourth intercostal space thoracotomy was performed, and the pericardium was excised to expose the heart. The left anterior descending coronary artery (LAD) was ligated $2 \mathrm{~mm}$ above the left auricle by a $6-0$ silk suture to induce regional myocardial ischemia. After $30 \mathrm{~min}$ of ischemia, the ligature was loosened to allow reperfusion for $2 \mathrm{~h}$. Sham-operated rats underwent the same surgical procedures, without tying the 6-0 silk suture. At the end of reperfusion, rats were killed, and parts of the anterior wall of the left ventricular myocardium near the cardiac apex and blood samples were obtained for further analysis.

2.5. Experimental Protocol. Eight weeks after STZ administration, rats were randomly allocated into 4 groups as follows: Group 1 rats (Sham), received vehicle (10 ml/kg saline) but no tightening of the coronary sutures, Group 2 rats $(\mathrm{I} / \mathrm{R})$, received vehicle $(10 \mathrm{ml} / \mathrm{kg}$ saline $)$ and were subjected to $30 \mathrm{~min}$ of ischemia followed by $2 \mathrm{~h}$ of reperfusion, Group 3 rats (SFI), treated with $10 \mathrm{ml} / \mathrm{kg}$ SFI and were subjected to $30 \mathrm{~min}$ of ischemia followed by reperfusion, Group 4 rats (SFI+WOR), treated with $10 \mathrm{ml} / \mathrm{kg}$ SFI plus a specific inhibitor of PI3K wortmannin and were subjected to $30 \mathrm{~min}$ of ischemia followed by reperfusion. Wortmannin $(15 \mathrm{ug} / \mathrm{kg})$ was administered $20 \mathrm{~min}$ before coronary ischemia and reperfusion, $10 \mathrm{~min}$ before the administration of SFI. The time course of the experiments is depicted in Figure 1. The dose of SFI and wortmannin were based on previous studies of myocardial ischemia-reperfusion injury $[7,8]$.

2.6. Hemodynamics. Hemodynamic measurements included heart rate (HR) and mean arterial pressure (MAP). The rate pressure product (RPP) was calculated as the product of the heart rate and the peak mean arterial pressure. These parameters were continuously measured during baseline, $30 \mathrm{~min}$ into ischemia and at $2 \mathrm{~h}$ of reperfusion.

2.7. Determination of Infarct Size. At the end of the 2-hourreperfusion period, the ligature around the coronary artery was retied again, and $2 \mathrm{ml}$ of $0.25 \%$ EB dye was injected into the aorta to map the normally perfused region of the heart. The myocardial area at risk (AAR) for infarction was delineated by the area of myocardium not dyed. The presence of EB was used to identify the area that was not subjected to the ischemia. Rat hearts were rapidly excised and frozen at -20 degree Celsius and then sliced into $2 \mathrm{~mm}$ thick sections perpendicular to the heart base-apex axis using a heart slice chamber. The slices were incubated in 1\% TTC in pH 7.4 buffer for $15 \mathrm{~min}$ at 37 degree Celsius to distinguish the viable myocardium from the necrotic. The viable tissue was stained red by TTC, while the infarct portion not taking up TTC stain remained pale. Morphometric measurements of the AAR and infarct area (IA) in each slice were performed with a scanner (Epson, v30, Japan) and an image analysis system (Image-Pro plus; Media Cybernetics, Bethesda, MA). The percentage of ratios of AAR versus left ventricle (LV) $(\mathrm{AAR} / \mathrm{LV})$ and IA versus AAR (IA/AAR) were calculated.

\subsection{Plasma Creatine Kinase Isoenzyme and Lactate Dehydro- genase Assay. Arterial blood samples were collected at the end of reperfusion and centrifuged at $3000 \mathrm{rpm}$, for $10 \mathrm{~min}$ at 4 degree Celsius. Two specific marker enzymes, including the creatine kinase isoenzyme (CK) and lactate dehydrogenase (LDH) from the plasma, were measured by using commercial kits (Beijing Kemeidongya Biotechnology Ltd., China). The content of these marker enzymes was expressed as U/L.}

2.9. Determination of Myocardial Apoptosis. Terminal deoxynucleotidyl nick-end labeling (TUNEL) assay was used to assess myocardial apoptosis with an apoptosis detection kit (Roche, Basel, Switzerland). TUNEL-positive cardiomyocytes in the ischemic myocardium were carefully evaluated under double-blind conditions. The percentage of TUNEL-positive cells was determined by dividing the number of positive-staining nuclei by the total number of nuclei of the cell.

2.10. Western Blot Analysis. Myocardium tissue samples $(100 \mathrm{mg})$ were lysed with lysis buffer. After sonication, the lysates were centrifuged, and protein concentration was determined with BCA protein assay kit (Beyotime Biotech Inc, Jiangsu, China). Protein extracts were separated by electrophoresis on SDS-PAGE and then transferred onto a polyvinylidene difluoride (PVDF) membrane. The membranes were incubated in 5\% dry milk for $1 \mathrm{~h}$ and then incubated with the following primary antibodies: phosphorylated Akt (p-Akt, Ser473), Akt, phosphorylated endothelial nitric oxide synthase (p-eNOS, Ser1177), eNOS, caspase-3 (Cell Signaling), Bcl-2, and glyceraldehyde-3phosphate dehydrogenase (GADPH, Santa Cruz). Subsequently, the membranes were washed and incubated with the 


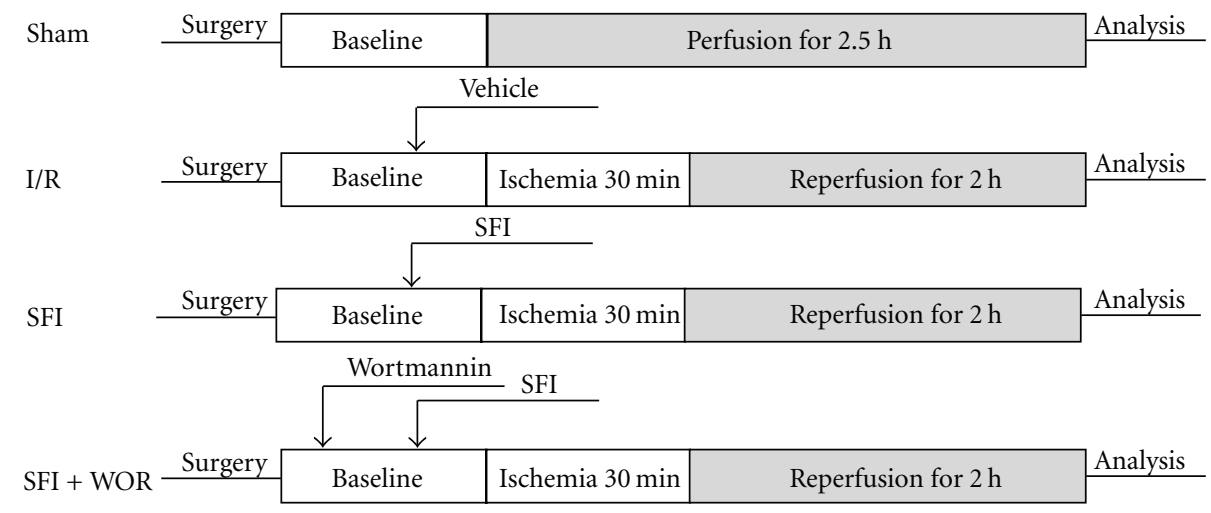

FIGURE 1: Experimental protocols. Diabetic rats were subjected to $30 \mathrm{~min}$ of coronary artery occlusion followed by $2 \mathrm{~h}$ of reperfusion. SFI was applied 10 minutes before ischemia. Wortmannin was applied 20 minutes prior to ischemia. I/R: ischemia/reperfusion; SFI: Shen-fu injection; WOR: wortmannin.

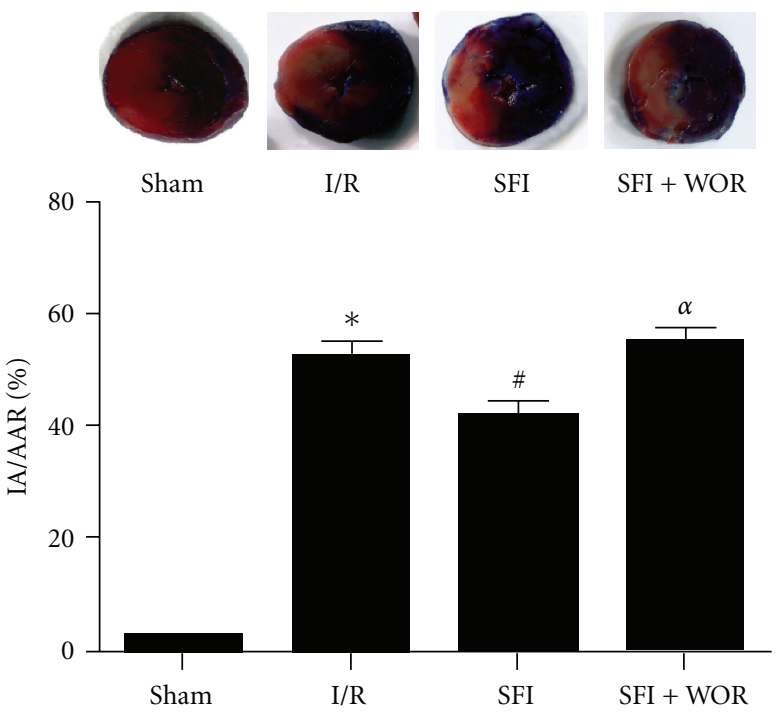

FIGURE 2: Myocardial infarct size in Sham-operated rats or in rats subjected to $30 \mathrm{~min}$ ischemia followed by $2 \mathrm{~h}$ of reperfusion. The blue-stained areas represent nonischemic tissue, and redstained areas represent the area at risk. Pale areas indicate infarct areas. Values presented are mean \pm SEM. AAR: area at risk; IA: infarct area; I/R: ischemia/reperfusion; SFI: Shen-fu injection; WOR: wortmannin. $n=6,{ }^{*} P<.05$ versus Sham, ${ }^{\#} P<.05$ versus $\mathrm{I} / \mathrm{R}$, and ${ }^{\alpha} P<.05$ versus SFI.

corresponding horseradish peroxidase-conjugated secondary antibody. The protein bands were visualized with ECL plus reagent (Pierce Biotechnology Inc., Rockford, IL). GADPH was chosen as a loading control to further assure the same volume for all the samples.

\subsection{Activity of Superoxide Dismutase and the Level of Malon-} dialdehyde in Myocardial Tissues. At the end of reperfusion, the myocardial supernatant was isolated from ischemia heart tissue samples by centrifugation at $4000 \mathrm{rpm}$ for $10 \mathrm{~min}$ at $4{ }^{\circ} \mathrm{C}$. The superoxide dismutase (SOD) activity and malondialdehyde (MDA) level were measured by colorimetric analysis using a spectrophotometer with the associated detection kits (Jiancheng, Nanjing, China), respectively.

2.12. Statistical Analysis. All values are presented as mean \pm SEM. Differences between groups were determined by using one-way ANOVA Analysis followed by Bonferroni's post hoc test. A value of $P<.05$ was considered to be statistically significant.

\section{Results}

3.1. Basic Parameters. There were no major differences between groups in terms of blood glucose, body weight, and the heart to body weight ratio before the MI/R (Table 1).

3.2. Systemic Hemodynamics. There were no significant differences in HR, MAP, and RRP among groups during coronary artery occlusion and reperfusion.

3.3. Effect of SFI on Myocardial Infarct Size. To examine whether SFI treatment reduces the myocardial injury following $\mathrm{I} / \mathrm{R}$ in diabetic hearts, myocardial infarct size was measured. The representative images of AAR and IA from each group were shown in Figure 2. Thirty minutes of ischemia followed by $2 \mathrm{~h}$ of reperfusion resulted in significant myocardial infarction in $\mathrm{I} / \mathrm{R}$ rats compared with that in Sham-operated ones $(53.8 \pm 2.2 \%$ versus Sham, $P<.05)$. The infarct size was significantly decreased with the treatment of SFI $(42.4 \pm 6.7 \%$ versus I/R, $P<.05)$. Interestingly, administration of wortmannin abolished SFI-induced reduction in infarct size, and infarct was similar to I/R group (Figure 2). These results provided direct evidence that SFI reduces myocardial injury following I/R via PI3K/Akt-dependent signaling pathway. No significant difference in AAR was found between the four groups $(P>.05)$.

\subsection{Effect of SFI on Plasma CK and LDH Activities in Diabetic} Rats. To determine whether SFI may also reduce myocardial cellular damage induced by MI/R, the activities of CK and $\mathrm{LDH}$ that are indices of myocardial cellular injury were 


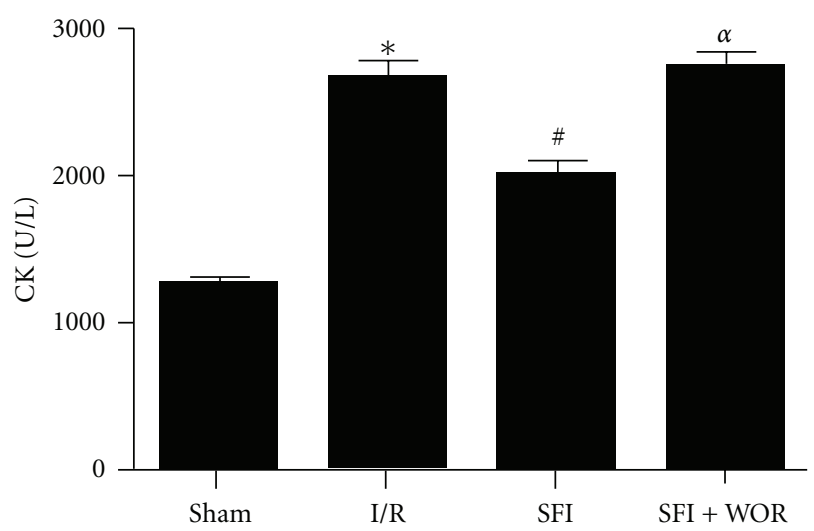

(a)

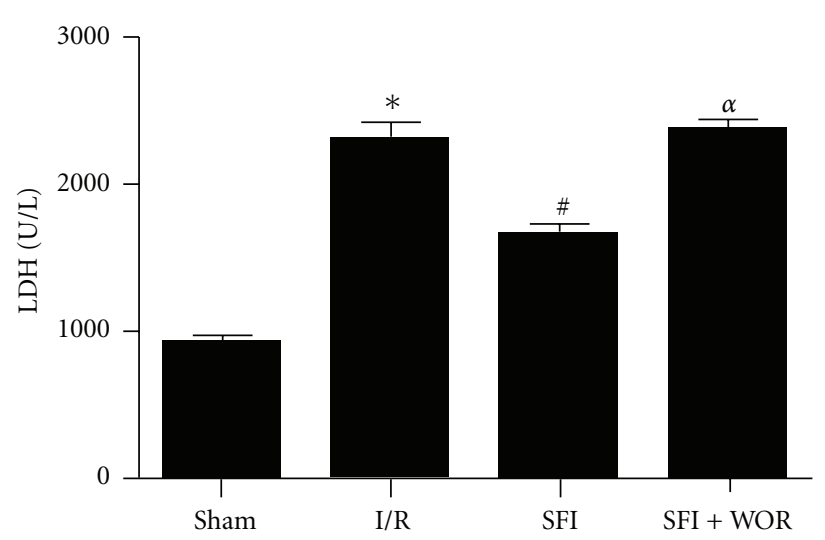

(b)

FIGURE 3: Plasma creatine kinase (CK) and lactate dehydrogenase (LDH). I/R: ischemia/reperfusion; SFI: Shen-fu injection; WOR: wortmannin. Values presented are mean \pm SEM. $n=8,{ }^{*} P<.05$ versus Sham, ${ }^{\#} P<.05$ versus I/R, and ${ }^{\alpha} P<.05$ versus SFI.

TABLE 1: Basic parameters of the rats.

\begin{tabular}{|c|c|c|c|c|c|}
\hline Basic parameters & Sham & $\mathrm{I} / \mathrm{R}$ & SFI & SFI+WOR & $P$ \\
\hline Blood glucose $(\mathrm{mmol} / \mathrm{l})$ & $22.8 \pm 1.1$ & $24.9 \pm 1.5$ & $24.3 \pm 1.4$ & $21.8 \pm 1.1$ & .32 \\
\hline Body weight (g) & $239.9 \pm 6.3$ & $224.5 \pm 9.7$ & $233.3 \pm 9.0$ & $243.1 \pm 7.9$ & .41 \\
\hline Heart to body weight ratio $(\mathrm{mg} / \mathrm{g})$ & $2.93 \pm 0.18$ & $3.13 \pm 0.12$ & $2.85 \pm 0.10$ & $3.10 \pm 0.09$ & .37 \\
\hline
\end{tabular}

Values presented are mean \pm SEM. I/R: ischemia/reperfusion; SFI: Shen-fu injection; WOR: wortmannin. $n=16$ in each group.
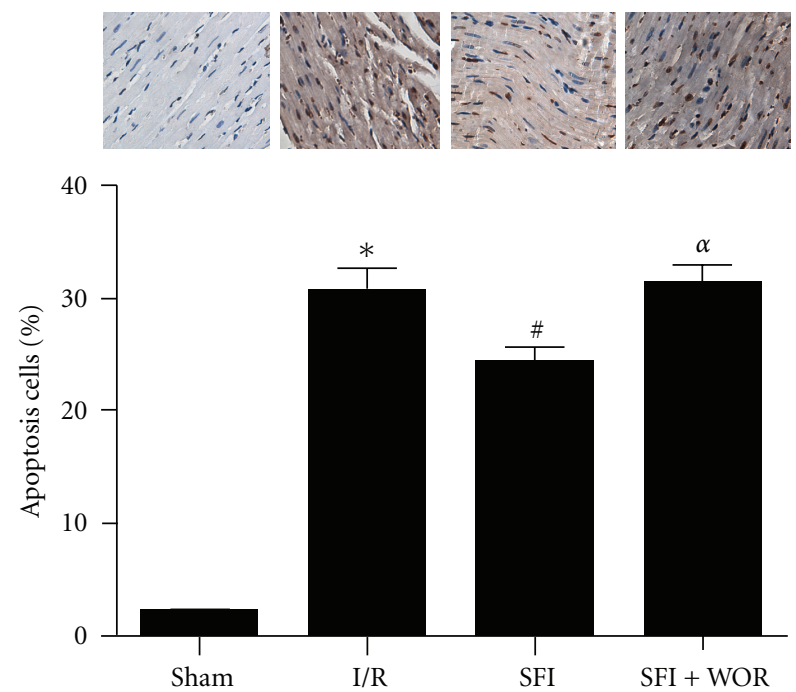

FIGURE 4: Representative photomicrographs of TUNEL staining from Sham, I/R, SFI, and SFI+WOR groups, respectively $(\times 400)$. I/R: ischemia/reperfusion; SFI: Shen-fu injection; WOR: wortmannin. Values presented are mean \pm SEM. $n=4,{ }^{*} P<.05$ versus Sham, ${ }^{\#} P<.05$ versus $\mathrm{I} / \mathrm{R}$, and ${ }^{\alpha} P<.05$ versus $\mathrm{SFI}$.

measured at the end of reperfusion. In comparison with Sham group, I/R caused a significant increase in CK and LDH (both having $P<.05$, Figure 3). However, $\mathrm{CK}$ and $\mathrm{LDH}$ activities were decreased in rats treated with SFI compared with $\mathrm{I} / \mathrm{R}$ group (both having $P<.05$ ). Pretreatment with wortmannin significantly blunted the SFI-induced decrease of plasma CK and LDH (both having $P<.05$ versus SFI group).

3.5. Effect of SFI on Myocardium Apoptosis in Diabetic Rats. As shown in Figure 4, the percentage of TUNEL-positive myocyte nuclei was significantly increased in the I/R group compared with the Sham group $(P<.05)$. SFI treatment reduced TUNEL-positive cells in hearts subjected to MI/R (versus I/R group, $P<.05$ ). Pretreatment of the rats with wortmannin attenuated the effect of SFI in reducing TUNELpositive cells.

3.6. Effect of SFI on the Protein Phosphorylation of Akt in Diabetic Rats. After $2 \mathrm{~h}$ of reperfusion, no significant changes were identified in total Akt expression among the Sham, I/R, SFI, and SFI+WOR groups (Figures 5(a) and 5(b)). Akt phosphorylation has been demonstrated to reflect Akt activation. Treatment with SFI significantly increased the expression of $\mathrm{p}$-Akt $(P<.05$, SFI versus $\mathrm{I} / \mathrm{R})$. In addition, the effect of SFI on Akt phosphorylation was completely abolished by treatment with wortmannin, a specific PI3K blocker $(P<.05$, SFI versus SFI+WOR) (Figure 5(c)).

3.7. Effect of SFI on the Protein Phosphorylation of eNOS in Diabetic Rats. It has been reported that eNOS is a substrate for Akt and NO production following Akt-evoked eNOS phosphorylation [9]. We therefore attempted to determine whether eNOS phosphorylation contributes to SFI-induced cardioprotection. As illustrated in Figure 6, there was no significant difference in eNOS expression among different groups, while treatment with SFI resulted in significant 


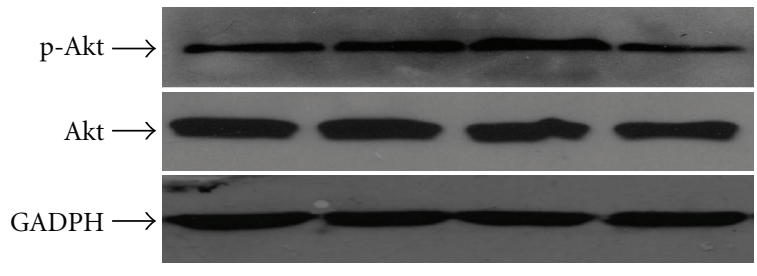

(a)

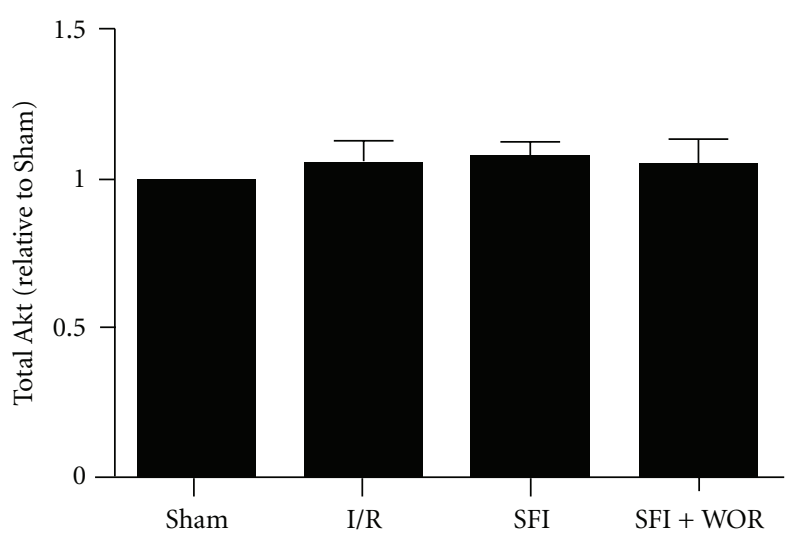

(b)

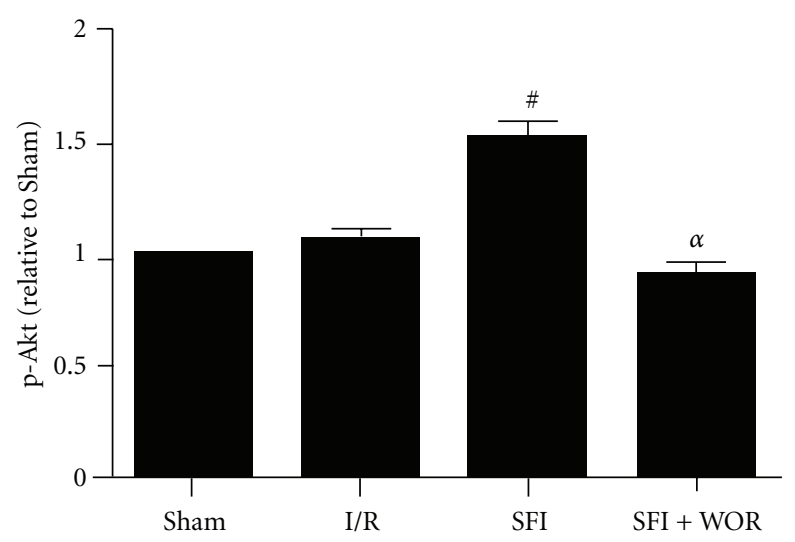

(c)

FIGURE 5: Immunoblots and graphs showing the protein levels and fold increases in total and phosphorylated Akt from Sham, I/R, SFI, and SFI+WOR groups, respectively. I/R: ischemia/reperfusion; SFI: Shen-fu injection; WOR: wortmannin. Values presented are mean \pm SEM. $n=6,{ }^{\#} P<.05$ versus $\mathrm{I} / \mathrm{R}$, and ${ }^{\alpha} P<.05$ versus $\mathrm{SFI}$.

expression of $\mathrm{p}$-eNOS $(P<.05, \mathrm{I} / \mathrm{R}$ versus SFI). Most importantly, to explore whether the increase in $\mathrm{p}$-eNOS was related to PI3K/Akt pathway, diabetic rats were pretreated with wortmannin. The results showed that treatment with wortmannin completely abrogated the increase in p-eNOS $(P<.05$, SFI versus SFI+WOR $)$.

3.8. Effect of SFI on Bcl-2 and Caspase-3 Cleavage in Diabetic Rats. We measured antiapoptotic protein Bcl-2 and proapoptotic protein caspase-3 expression in the present study. As shown in Figure 7, SFI enhanced the expression of $\mathrm{Bcl}-2$ compared to the saline-treated I/R rats. Pretreatment with wortmannin abolished the effect of SFI-induced Bcl2 upregulation and decreased it to a level similar to the I/R group.

Caspase- 3 is a pivotal mediator of apoptosis, and myocardial caspase- 3 activity is suggested as a marker of MI/R myocyte apoptosis [10]. Caspase-3 normally exists as a $32-$ $\mathrm{kDa}$ inactive precursor that is cleaved proteolytically to an active p17 subunit when cells are induced to undergo apoptosis. To determine whether caspase- 3 was activated, we detected the cleaved (activate) caspase- 3 protein by Western blotting. As shown in Figure 7, there were no changes in total (inactivate) caspase- 3 proteins among the 4 groups.
The activate caspase- 3 was significantly increased after MI/R as compared with that in Sham group (I/R versus Sham, $P<.05)$. The activate caspase- 3 was decreased in SFI group (Figure $7(\mathrm{~d}), P<.05, \mathrm{I} / \mathrm{R}$ versus $\mathrm{SFI}$ ), and pretreatment with wortmannin abolished the caspase-3 activation.

3.9. Effect of SFI on Activity of SOD and Level of MDA in Diabetic Rats. Data are shown in Figure 8. Compared with the Sham group, the I/R-induced injury was manifested by a significant increase in MDA and decrease in SOD. The administration of SFI caused a significant decrease in the MDA content and increase in SOD enzyme activity compared with the I/R group. Moreover, SFI-induced changes in MDA and SOD were reverted by wortmannin treatment.

\section{Discussion}

Several important observations were made in the present study. First, we have shown that SFI treatment attenuated $\mathrm{MI} / \mathrm{R}$ injury in diabetic rats as evidenced by attenuated myocardial apoptosis and reduced infarct size in a PI3K/Aktdependent manner. Second, Akt-evoked eNOS phosphorylation significantly contributes to SFI-induced cardioprotection. In addition, we have provided evidence that SFI protects 


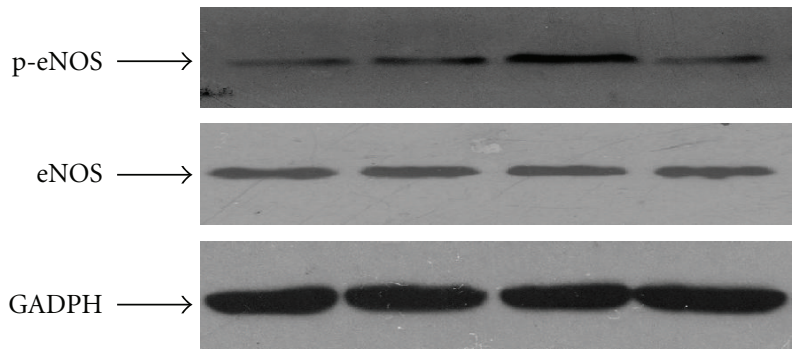

(a)

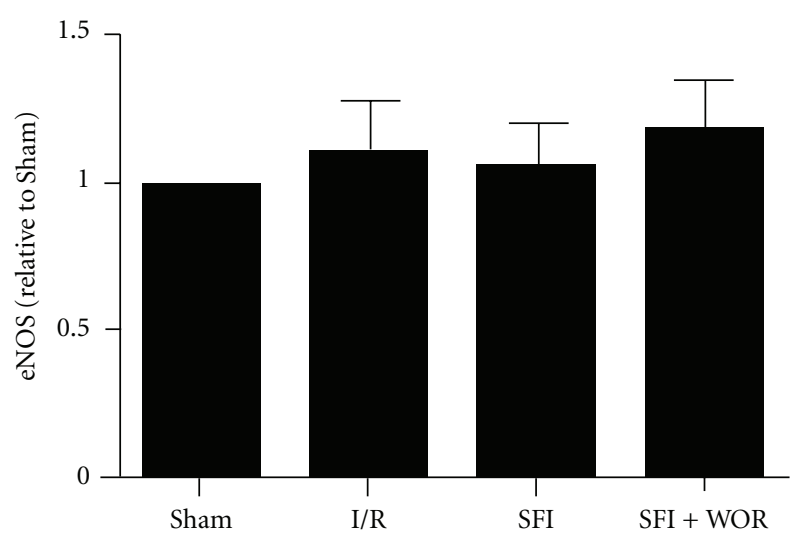

(b)

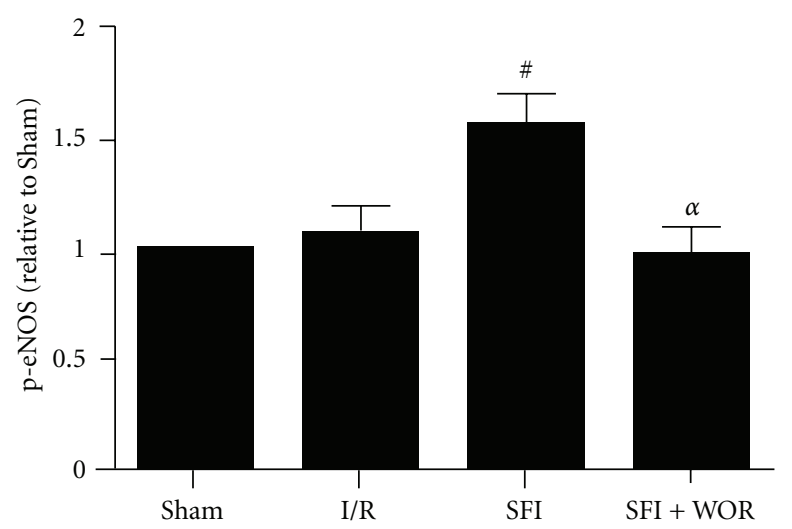

(c)

FIGURE 6: Immunoblots and graphs showing the protein levels and fold increases in total and phosphorylated eNOS from Sham, I/R, SFI, and SFI+WOR groups, respectively. I/R: ischemia/reperfusion; SFI: Shen-fu injection; WOR: wortmannin. Values presented are mean \pm SEM. $n=6,{ }^{\#} P<.05$ versus $\mathrm{I} / \mathrm{R}$, and ${ }^{\alpha} P<.05$ versus SFI.

the diabetic hearts from oxidative stress even in the absence of ischemia/reperfusion.

Shen-fu injection is composed of Ginseng and Fuzi. Previous data from experimental studies have demonstrated that SFI has significant protective effects on cardiovascular system. Zheng et al. studied myocardial infarction and reperfusion injury in an in vivo rat model and discovered that SFI reduced the size of infarction and improved the pathologic changes of myocardium during ischemia reperfusion [8]. In cell culture model, Wang et al. reported that SFI can prevent cardiomyocytes apoptosis induced by hypoxia/reoxygenation injury via upregulation of $\mathrm{Bcl}-2$ protein levels and downregulation of caspase-3 [11]. One of our recent studies showed that the SFI reduced MI/R injury in congenital heart patients also supported these conclusions.The SFI-induced inactivation of caspase- 3 may lead to inhibition of myocardial apoptosis after MI/R. It was, therefore, important to study whether SFI can also afford similar cardioprotection in hearts from diabetic subjects.

Among numerous signaling pathways involved in regulation of cell survival, PI3K/Akt signaling pathway plays a crucial role in protecting the myocardium from MI/R injury [12-14]. Previous studies have demonstrated that pharmacologic preconditioning fails to induce cardioprotection in
STZ-induced diabetic animals, and PI3K/Akt pathway was impaired in diabetic rats [15-17]. In our present study, we detected significant activation of survival pathway as evidenced by robust phosphorylation of Akt in SFI-treated diabetic myocardium following ischemia reperfusion. However, expression of p-Akt was undetectable when wortmannin was given along with SFI therapy. It seems that SFI induces cardioprotective effects through the activation of PI3K/Akt pathway in diabetic rats. The protein expression of PI3K was not assessed in the current study. Further investigation will be needed to explore whether SFI act directly on upstream signaling pathways.

How activation of Akt increases cardiomyocyte resistance to $\mathrm{MI} / \mathrm{R}$ injury in diabetic rats is incompletely understood. Strong evidence shows that eNOS is an important target of Akt and eNOS functions as an important cardiovascular protective molecule $[9,18,19]$. Recent studies show that increased expression of p-Akt and p-eNOS alleviates the IR injury in the diabetic myocardium [17, 19]. Our study showed that SFI treatment resulted in an enhanced level peNOS which was significantly higher than that of salinetreated I/R group. These results suggest that SFI-induced cardioprotective effects are mediated through Akt-induced eNOS phosphorylation. eNOS is the source of NO and is 


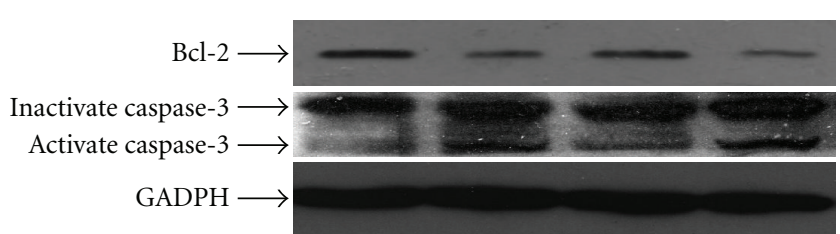

(a)

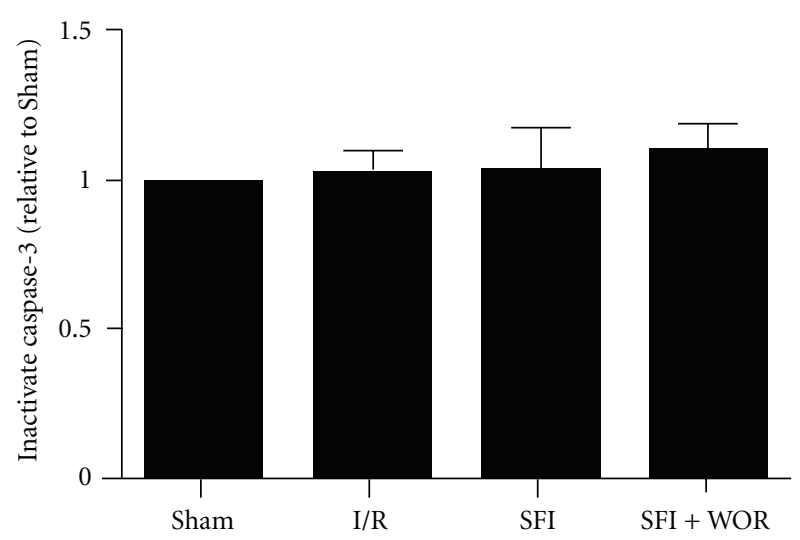

(c)

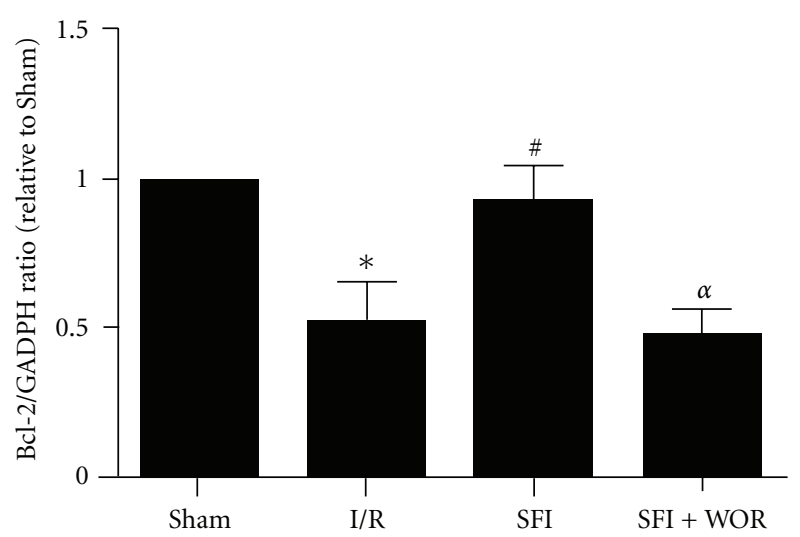

(b)

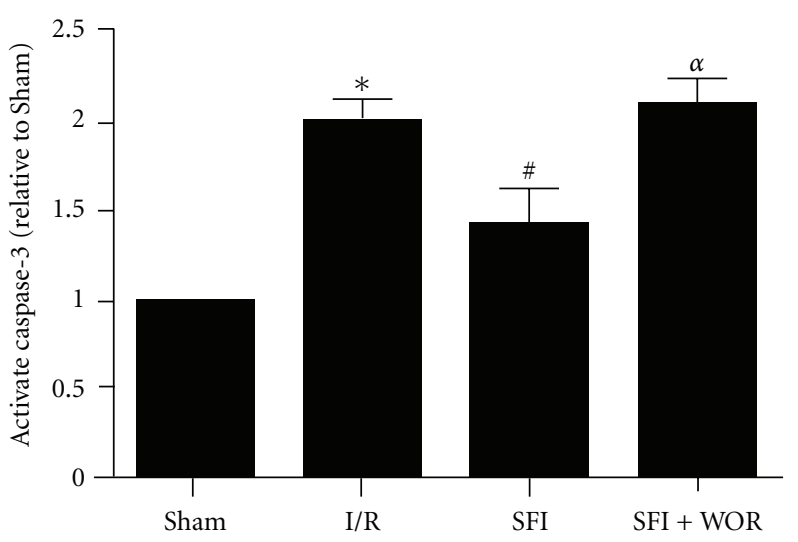

(d)

FiguRE 7: Immunoblots and graphs showing the protein levels of bcl-2 and caspase-3 from Sham, I/R, SFI, and SFI+WOR groups, respectively. I/R: ischemia/reperfusion; SFI: Shen-fu injection; WOR: wortmannin. Values presented are mean \pm SEM. $n=6,{ }^{*} P<.05$ versus Sham, ${ }^{\#} P<.05$ versus $\mathrm{I} / \mathrm{R}$, and ${ }^{\alpha} P<.05$ versus $\mathrm{SFI}$.

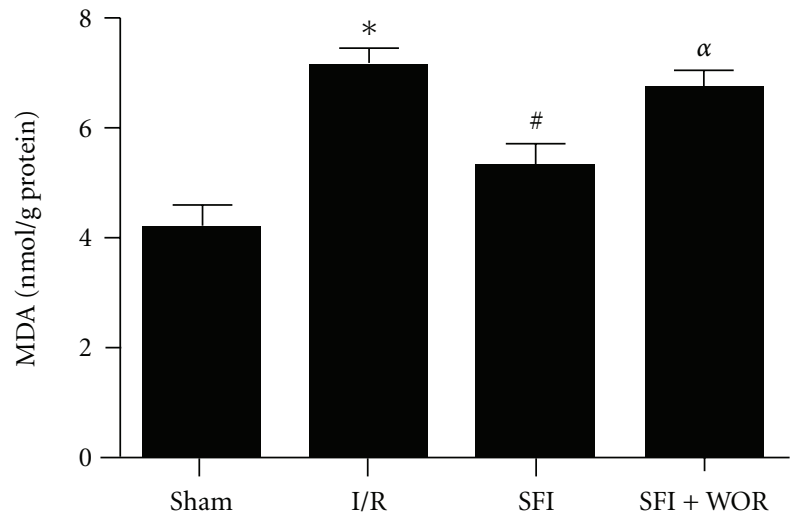

(a)

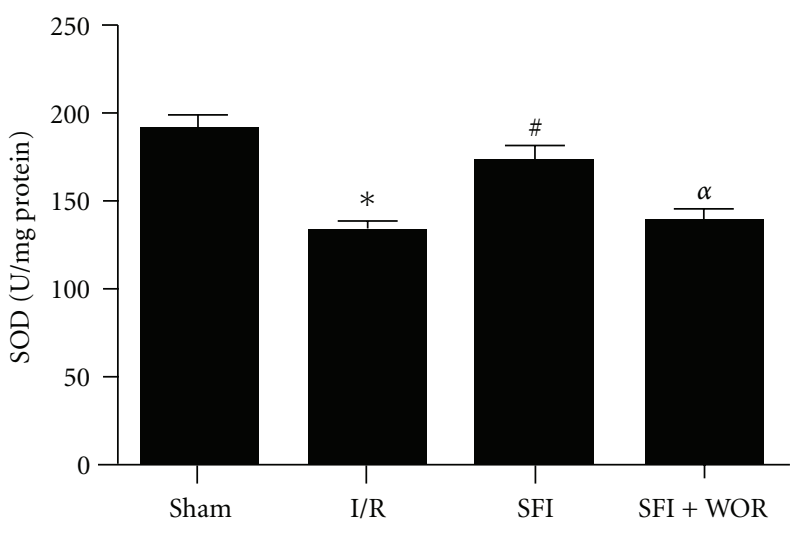

(b)

FIGURE 8: Malondialdehyde (MDA) and superoxide dismutase (SOD). I/R: ischemia/reperfusion; SFI: Shen-fu injection; WOR: wortmannin. Values presented are mean \pm SEM. $n=6,{ }^{*} P<.05$ versus Sham, ${ }^{\#} P<.05$ versus $\mathrm{I} / \mathrm{R}$, and ${ }^{\alpha} P<.05$ versus SFI.

important in regulation of vasodilatation, vascular remodeling, cardiac structure, and angiogenesis [20, 21]. It has been shown that $\mathrm{NO}$ inhibits apoptosis both in vitro and in vivo $[9,22]$. $\mathrm{NO}$ can rapidly elevate $\mathrm{Bcl}-2$ expression at protein and mRNA level [23-25]. The level of Bcl-2 protein has been suggested to determine cardiac myocyte survival or death after ischemia and reperfusion [9, 25-27]. The release of $\mathrm{Bcl}-2$ during ischemia-reperfusion injury has been proven to prevent apoptosis by blocking the release of cytochrome $c$ from the mitochondria and ameliorating the activation 
of caspase-3 $[25,28]$. Caspase- 3 is an intracellular cysteine protease that is one of the key executioners of apoptosis $[8,29,30]$. As expected, pretreatment with SFI significantly inhibited the activation of subsequent effector caspase-3. The SFI-induced inactivation of caspase-3 may lead to its inhibition of myocardial apoptosis after MI/R.

On the other hand, numerous studies have shown that the production of reactive oxygen species (ROS) is increased in diabetes. Excessive ROS leads to inactivation of $\mathrm{NO}$ and thus impaired NO-dependent cardioprotection [3133]. MDA is a marker of lipid peroxidation that occurs as a result of the damaging effects of ROS, and SOD is a cellular ROS scavenger $[34,35]$. In our study, following SFI treatment, we found that the increase of SOD activity and the decrease of MDA production may have provided extensive protection to diabetic hearts and facilitated NO-dependent cardioprotection. Considerable evidence demonstrates that SFI has significant protective effects in different organs. Qian et al. reported that SFI inhibited endotoxin-induced pulmonary inflammation in vivo [36]. SFI is also reported to have protective effect on gastrointestinal microcirculation after myocardial IR injury in rabbits, in a dose-independent manner [37]. Furthermore, SFI can inhibit the production of oxygen free radical and decrease apoptosis of liver cell in a liver graft model [38].

There are several limitations with the present study that should be mentioned. First, the cardiac protective effect of SFI in nondiabetic rats was not investigated in our study. It is reported that SFI can protect normal rats from MIR injury, with the increase of SOD and decrease of the MDA contents [8]. Therefore, it seems that SFI has similar beneficial effect to both nondiabetic and diabetic rats. Second, we did not directly measure the cardiac function during the experiment as the primary goal of the study is to explore the signaling pathway that may involve Shen-fu cardioprotection. However, we have collected hemodynamic parameters, including HR, MAP, and RPP, which indirectly indicate that SFI has no major effect on cardiac function. Third, the amount of NO was not monitored in this study. NO is produced from eNOS, neuronal NOS (nNOS), and inducible NOS (iNOS) in the heart. Further study will be needed to explore whether Shen-Fu injection can modulate the NO production as well as the underling mechanism.

In summary, the current study demonstrated that SFI could protect cardiomyocytes against MI/R injury and inhibits apoptosis in diabetic rats via activating PI3K/Akt/eNOS signaling pathway.

\section{Acknowledgment}

This work was supported by the National Natural Science Foundation of China (no. 30672033).

\section{References}

[1] S. M. Donahoe, G. C. Stewart, C. H. McCabe et al., "Diabetes and mortality following acute coronary syndromes," Journal of the American Medical Association, vol. 298, no. 7, pp. 765-775, 2007.
[2] S. Boudina and E. D. Abel, "Diabetic cardiomyopathy revisited," Circulation, vol. 115, no. 25, pp. 3213-3223, 2007.

[3] D. Aronson and E. R. Edelman, "Revascularization for coronary artery disease in diabetes mellitus: angioplasty, stents and coronary artery bypass grafting," Reviews in Endocrine and Metabolic Disorders, vol. 11, no. 1, pp. 75-86, 2010.

[4] T. F. Lüscher, M. A. Creager, J. A. Beckman, and F. Cosentino, "Diabetes and vascular disease. Pathophysiology, clinical consequences, and medical therapy: part II," Circulation, vol. 108, no. 13, pp. 1655-1661, 2003.

[5] J. Herlitz, B. W. Karlson, J. Lindqvist, and M. Sjölin, "Rate and mode of death during five years of follow-up among patients with acute chest pain with and without a history of diabetes mellitus," Diabetic Medicine, vol. 15, no. 4, pp. 308-314, 1998.

[6] Z.-Y. Xia, X.-Y. Liu, L.-Y. Zhan, Y.-H. He, T. Luo, and Z. Xia, "Ginsenosides compound (shen-fu) attenuates gastrointestinal injury and inhibits inflammatory response after cardiopulmonary bypass in patients with congenital heart disease," Journal of Thoracic and Cardiovascular Surgery, vol. 130, no. 2, pp. 258-264, 2005.

[7] A. A. Bulhak, C. Jung, C.-G. Östenson, J. O. Lundberg, P.-O. Sjoquist, and J. Pernow, "PPAR- $\alpha$ activation protects the type 2 diabetic myocardium against ischemia-reperfusion injury: involvement of the PI3-kinase/Akt and NO pathway," American Journal of Physiology, vol. 296, no. 3, pp. H719H727, 2009.

[8] S.-Y. Zheng, J. Sun, X. Zhao, and J.-G. Xu, "Protective effect of Shen-Fu on myocardial ischemia-reperfusion injury in rats," American Journal of Chinese Medicine, vol. 32, no. 2, pp. 209220, 2004.

[9] F. Gao, E. Gao, T.-L. Yue et al., "Nitric oxide mediates the antiapoptotic effect of insulin in myocardial ischemiareperfusion: the roles of PI3-kinase, Akt, and endothelial nitric oxide synthase phosphorylation," Circulation, vol. 105, no. 12, pp. 1497-1502, 2002.

[10] L. Ji, F. Fu, L. Zhang et al., "Insulin attenuates myocardial ischemia/reperfusion injury via reducing oxidative/nitrative stress," American Journal of Physiology, vol. 298, no. 4, pp. E871-E880, 2010.

[11] Y.-L. Wang, C.-Y. Wang, B.-J. Zhang, and Z.-Z. Zhang, "Shenfu injection suppresses apoptosis by regulation of Bcl-2 and caspase-3 during hypoxia/reoxygenation in neonatal rat cardiomyocytes in vitro," Molecular Biology Reports, vol. 36, no. 2, pp. 365-370, 2009.

[12] Y. Zhang, L. Wei, D. Sun et al., "Tanshinone IIA pretreatment protects myocardium against ischaemia/reperfusion injury through the phosphatidylinositol 3-kinase/Akt-dependent pathway in diabetic rats," Diabetes, Obesity \& Metabolism, vol. 12, no. 4, pp. 316-322, 2010.

[13] P. H. Sugden, "Ras, Akt, and Mechanotransduction in the Cardiac Myocyte," Circulation Research, vol. 93, no. 12, pp. 1179-1192, 2003.

[14] B. D. Manning and L. C. Cantley, "AKT/PKB signaling: navigating downstream," Cell, vol. 129, no. 7, pp. 1261-1274, 2007.

[15] H. S. Kim, J. E. Cho, K. C. Hwang, Y. H. Shim, J. H. Lee, and Y. L. Kwak, "Diabetes mellitus mitigates cardioprotective effects of remifentanil preconditioning in ischemia-reperfused rat heart in association with anti-apoptotic pathways of survival," European Journal of Pharmacology, vol. 628, no. 1-3, pp. 132$139,2010$.

[16] E. R. Gross, A. K. Hsu, and G. J. Gross, "Diabetes abolishes morphine-induced cardioprotection via multiple pathways 
upstream of glycogen synthase kinase-3 $\beta$,' Diabetes, vol. 56, no. 1, pp. 127-136, 2007.

[17] S. V. Penumathsa, M. Thirunavukkarasu, S. M. Samuel et al., "Niacin bound chromium treatment induces myocardial Glut-4 translocation and caveolar interaction via Akt, AMPK and eNOS phosphorylation in streptozotocin induced diabetic rats after ischemia-reperfusion injury," Biochimica et Biophysica Acta, vol. 1792, no. 1, pp. 39-48, 2009.

[18] R. Schulz, M. Kelm, and G. Heusch, "Nitric oxide in myocardial ischemia/reperfusion injury," Cardiovascular Research, vol. 61, no. 3, pp. 402-413, 2004.

[19] M. Thirunavukkarasu, S. V. Penumathsa, S. Koneru et al., "Resveratrol alleviates cardiac dysfunction in streptozotocininduced diabetes: role of nitric oxide, thioredoxin, and heme oxygenase," Free Radical Biology and Medicine, vol. 43, no. 5, pp. 720-729, 2007.

[20] W. Xing, W. Yan, F. Fu et al., "Insulin inhibits myocardial ischemia-induced apoptosis and alleviates chronic adverse changes in post-ischemic cardiac structure and function," Apoptosis, vol. 14, no. 9, pp. 1050-1060, 2009.

[21] H. Takano, S. Manchikalapudi, X.-L. Tang et al., "Nitric oxide synthase is the mediator of late preconditioning against myocardial infarction in conscious rabbits," Circulation, vol. 98, no. 5, pp. 441-449, 1998.

[22] Y.-M. Kim, C. A. Bombeck, and T. R. Billiar, "Nitric oxide as a bifunctional regulator of apoptosis," Circulation Research, vol. 84, no. 3, pp. 253-256, 1999.

[23] C.-H. Yeh, Y.-M. Lin, Y.-C. Wu, Y.-C. Wang, and P. J. Lin, "Nitric oxide attenuates cardiomyocytic apoptosis via diminished mitochondrial complex I up-regulation from cardiac ischemia-reperfusion injury under cardiopulmonary bypass," Journal of Thoracic and Cardiovascular Surgery, vol. 128, no. 2, pp. 180-188, 2004.

[24] A. Das, L. Xi, and R. C. Kukreja, "Phosphodiesterase5 inhibitor sildenafil preconditions adult cardiac myocytes against necrosis and apoptosis: essential role of nitric oxide signaling," Journal of Biological Chemistry, vol. 280, no. 13, pp. 12944-12955, 2005.

[25] Y.-M. Kim, T.-H. Kim, D.-W. Seol, R. V. Talanian, and T. R. Billiar, "Nitric oxide suppression of apoptosis occurs in association with an inhibition of Bcl-2 cleavage and cytochrome c release," Journal of Biological Chemistry, vol. 273, no. 47, pp. 31437-31441, 1998.

[26] J. Raphael, S. Abedat, J. Rivo et al., "Volatile anesthetic preconditioning attenuates myocardial apoptosis in rabbits after regional ischemia and reperfusion via Akt signaling and modulation of Bcl-2 family proteins," Journal of Pharmacology and Experimental Therapeutics, vol. 318, no. 1, pp. 186-194, 2006.

[27] J. Feng, C. Bianchi, J. L. Sandmeyer, J. Li, and F. W. Sellke, "Molecular indices of apoptosis after intermittent blood and crystalloid cardioplegia," Circulation, vol. 112, supplement 9, pp. 184-189, 2005.

[28] C. S. Boyd and E. Cadenas, "Nitric oxide and cell signaling pathways in mitochondrial-dependent apoptosis," Biological Chemistry, vol. 383, no. 3-4, pp. 411-423, 2002.

[29] W. C. Earnshaw, L. M. Martins, and S. H. Kaufmann, "Mammalian caspases: structure, activation, substrates, and functions during apoptosis," Annual Review of Biochemistry, vol. 68, pp. 383-424, 1999.

[30] G. Ma, M. Al-Shabrawey, J. A. Johnson et al., "Protection against myocardial ischemia/reperfusion injury by shortterm diabetes: enhancement of VEGF formation, capillary density, and activation of cell survival signaling," NaunynSchmiedeberg's Archives of Pharmacology, vol. 373, no. 6, pp. 415-427, 2006.

[31] L. R. La Bonte, G. Davis-Gorman, G. L. Stahl, and P. F. McDonagh, "Complement inhibition reduces injury in the type 2 diabetic heart following ischemia and reperfusion," American Journal of Physiology, vol. 294, no. 3, pp. H1282H1290, 2008.

[32] J. Matejíková, J. Kucharská, D. Pancza, and T. Ravingerová, "The effect of antioxidant treatment and NOS inhibition on the incidence of ischemia-induced arrhythmias in the diabetic rat heart," Physiological Research, vol. 57, supplement 2, pp. S55-S60, 2008.

[33] D. Jay, H. Hitomi, and K. K. Griendling, "Oxidative stress and diabetic cardiovascular complications," Free Radical Biology and Medicine, vol. 40, no. 2, pp. 183-192, 2006.

[34] F.-F. Gao, Q.-Y. Jia, F.-X. Guo et al., "Egr-1, a central and unifying role in cardioprotection from ischemia-reperfusion injury?" Cellular Physiology and Biochemistry, vol. 24, no. 5-6, pp. 519-526, 2009.

[35] J. Lu, D.-M. Wu, Y.-L. Zheng, B. Hu, and Z.-F. Zhang, "Purple sweet potato color alleviates $\mathrm{D}$-galactose-induced brain aging in old mice by promoting survival of neurons via PI3K pathway and inhibiting cytochrome C-mediated apoptosis," Brain Pathology, vol. 20, no. 3, pp. 598-612, 2010.

[36] Y. Qian, J. Sun, Z. Wang, and J. Yang, "Shen-Fu attenuates endotoxin-induced acute lung injury in rats," American Journal of Chinese Medicine, vol. 34, no. 4, pp. 613-621, 2006.

[37] X.-J. Zhang, L. Song, Z.-G. Zhou, and X.-M. Wang, "Effect of shenfu injection on gastrointestinal microcirculation in rabbits after myocardial ischemia-reperfusion injury," World Journal of Gastroenterology, vol. 12, no. 27, pp. 4389-4391, 2006.

[38] W.-H. Zhu, X.-S. Leng, and J.-Y. Zhu, "Effect of Shenfu injection on ischemia-reperfusion injury of rat liver graft," Hepatobiliary and Pancreatic Diseases International, vol. 5, no. 2, pp. 205-209, 2006. 


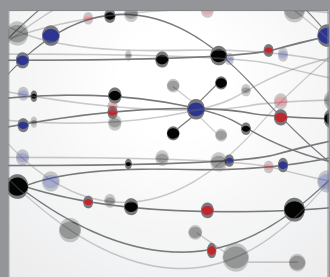

The Scientific World Journal
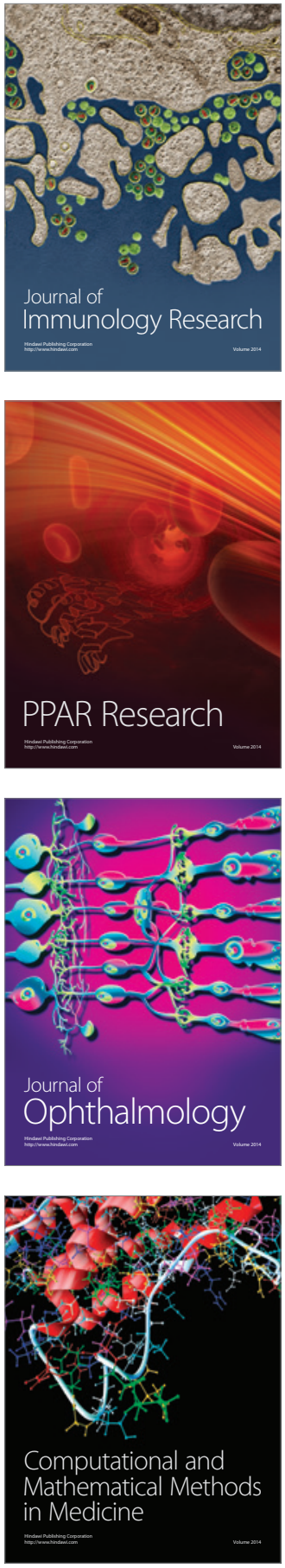

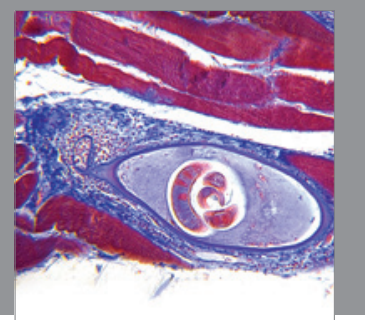

Gastroenterology

Research and Practice
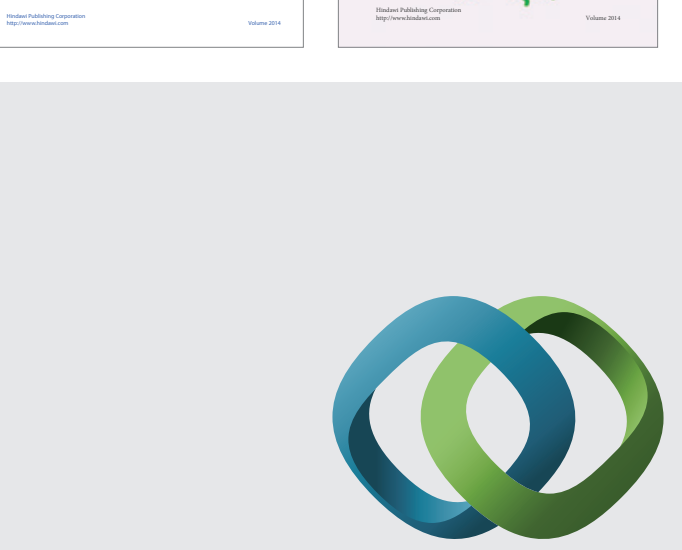

\section{Hindawi}

Submit your manuscripts at

http://www.hindawi.com
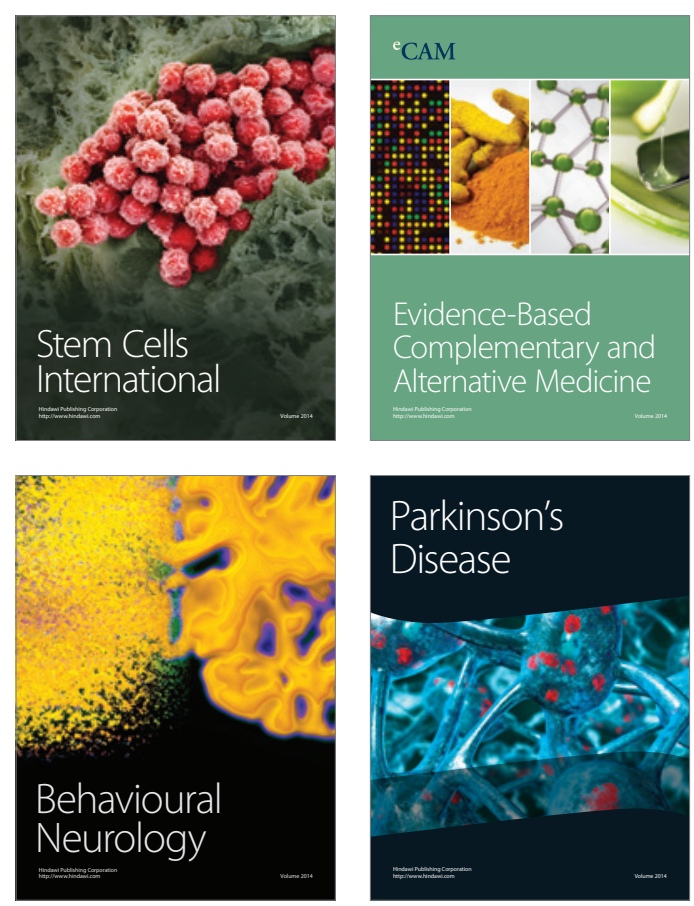

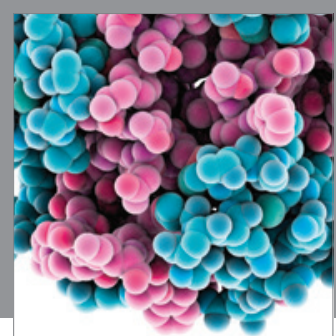

Journal of
Diabetes Research

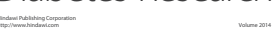

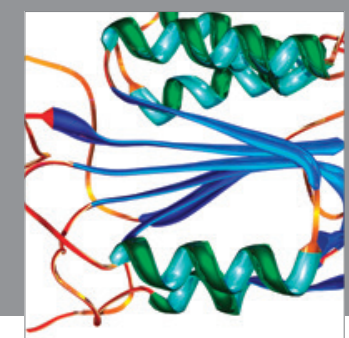

Disease Markers
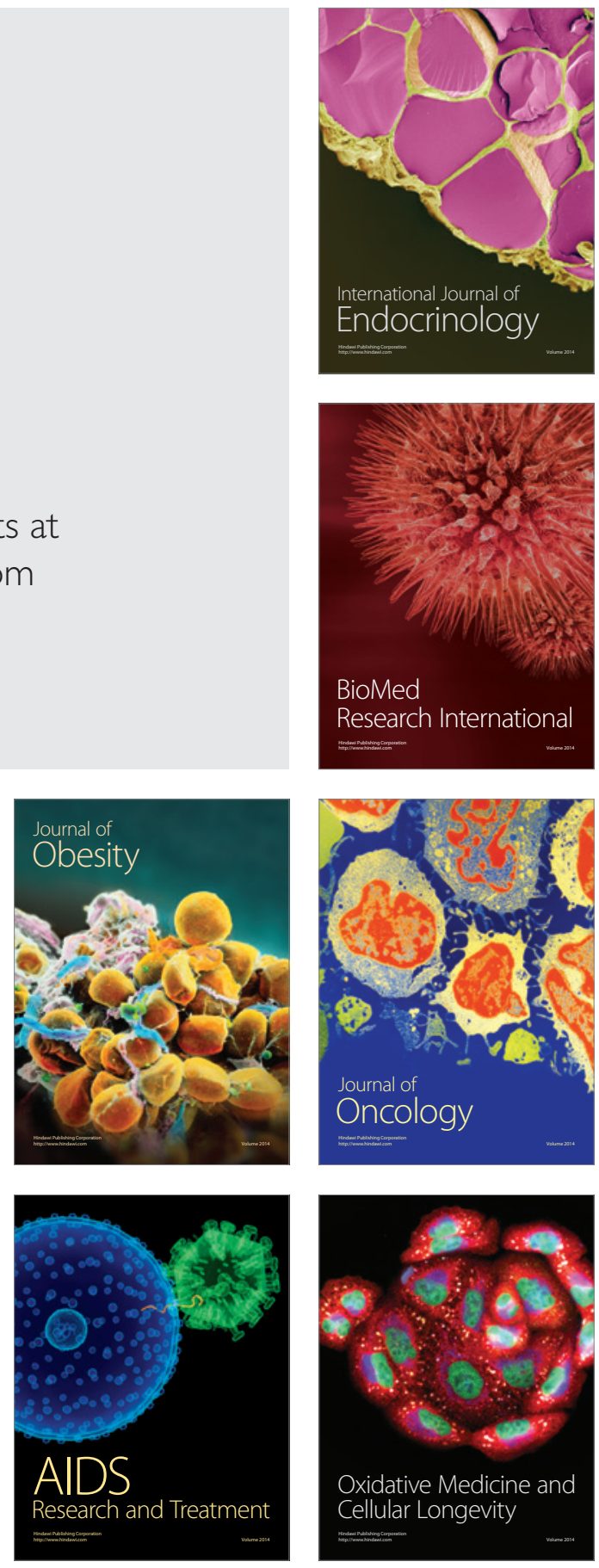